\title{
Sentiment Analyzing by Dictionary based Approach
}

\author{
Fatehjeet Kaur Chopra \\ Department of Computer Science \\ Punjabi University Regional Centre for Information \\ Technology and Management, \\ Mohali, Punjab, India
}

\author{
Rekha Bhatia, PhD \\ Department of Computer Science \\ Punjabi University Regional Centre for Information \\ Technology and Management, \\ Mohali, Punjab, India
}

\begin{abstract}
Sentiment analysis has emerged as a field of study since the widespread of World Wide Web and internet. Opinion refers to withdrawal of lines or phrase in the unprocessed and immense data which express an opinion. Sentiment analysis further recognizes the polarity of the viewpoint being extricated. In this paper it is proposed that the sentiment analysis done by dictionary based approach. The consequence viewpoint is described as very high, high, moderate, low and very low.
\end{abstract}

\section{Keywords}

Sentiment Analysis, Punjabi Language, Linguistic Resources.

\section{INTRODUCTION}

Sentiment Analysis [2] manages examining feelings, sentiments, and the intellect or mind of an author or a speaker in distinction to a specified bit of text. Sentiment analysis or opinion mining is a multidisciplinary and multifaceted artificial intelligence issue. Its point is to minimize the hole in the middle of human and PC. In this way, it is gathering of human insight and electronic knowledge for mining the content and arranging client notions, likes, despises and wishes.

Sentiment analysis involves categorizing viewpoints in text within categories such as "positive" or "negative" usually with an implied class of "neutral". Sentiment analysis is additionally termed as opinion mining or sound of the customer. Sentiment analysis seeks to identify the opinions prime a text interval such as an application is classifying a movie review as "thumbs up"(an indication of satisfaction) or "thumbs down"(an indication of disapproval). A unique knowledge engineering method is proposed to determine this sentiment polarity, that applies text-categorization methods to justify the subjective sections of the record or document. Withdrawing these sections can be executed using efficient methods for discovering minimum cuts in graphs and it remarkably aids fusion of cross-sentence contextual limitations.

\section{GENERAL ARCHITECTURE OF SENTIMENT ANALYSIS}

Review of a general architecture of a generic Sentiment Analysis(SA) system. The Sentiment Analysis System architecture is shown in Figure 1.

- The input feed into the sentiment analysis system is a corpus(collection) of documents(or records) in any format such as PDF, HTML, XML, and Word, among others.

- The documents in the corpus are then transformed to text and then their pre-processing occurs using a variety of linguistic tools such as stemming, tokenization, piece of speech tagging, entity withdrawal(extraction), and relation withdrawal. Also the system or arrangement may use a set of lexicons and linguistic tools(resources).

- The chief component or part of the system is the document analysis module, which utilizes the linguistic tools to gloss the pre-processed records with sentiment annotations.

- The observations may be attached to whole documents (for document-formed sentiment), to individual sentences (for sentence-formed sentiment) or to specific features of entities (for aspect-formed sentiment). These observations are the outcome of the system and they may be represented to the user utilizing different varieties of visualization tools.

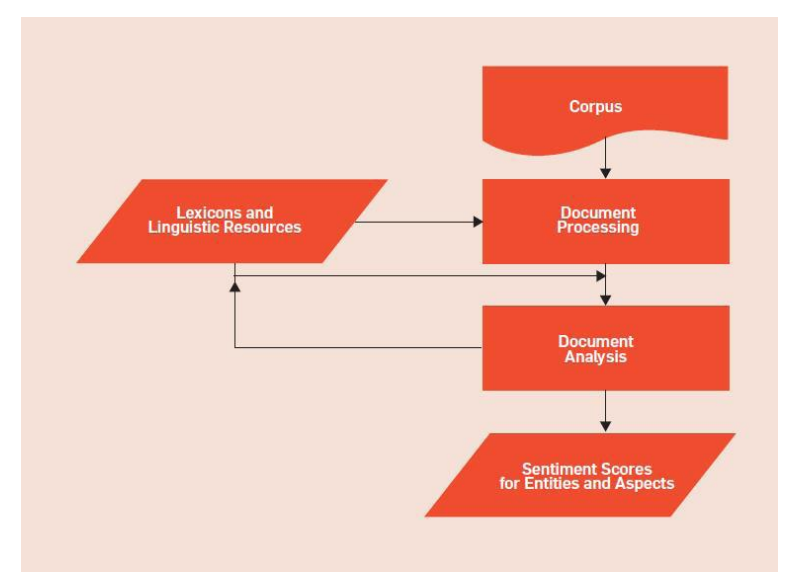

Figure 1. Architecture of Sentiment Analysis

\section{LITERATURE REVIEW}

Phyu Shein et al. [3] have shown a lot of text documents on the Web which contain opinions or sentiments about an object or article like product reviews, movies reviews, software reviews and book reviews and so on. Opinion mining or sentiment categorization goal is to withdraw the attributes on which viewers express their opinions and determine whether these are positive or negative. An ontology based combination approach to enhance the existing outlook of the sentiment categorization is proposed in this paper. The supervised learning techniques for classification of the sentiments inside software reviews is also utilized. The combination of using NLP(Natural Language Processing) techniques, ontology formed over Formal Concept Analysis (FCA) plan, and Support Vector Machine (SVM) for classifying the software reviews are positive, negative or neutral is proposed in this paper. Opinions are also important when someone before making a decision wants to hear other's viewpoints. Two types of opinions are : (1) Direct opinion and (2) Comparisons.

Direct opinions are the opinion expressing on the products, events, topics, and persons, etc. For example I am dismayed at the many versions of Windows7, and complete 90 
percent of the distinctions have nothing to do with me what so ever. Comparisons express the resemblance or differentiations between more than one thing such as the 2140 can run Windows 7 better than Windows Vista, and HP has always been good about participating in upgrade programs.

Joshi, A R et al. [4] have developed about a fall-back methodology to do supposition examination for Hindi reports, an issue on which, to the best of our insight, no work has been done as of not long ago. (A) First of all, three ways to deal with perform SA in Hindi is studied. A supposition explained corpora in the Hindi motion picture survey space have been built. The principal of the methodologies includes preparing a classifier on the clarified Hindi corpus and utilizing it to group another Hindi report. (B) In the second approach, an interpretation have been made of the given archive into English and a classifier prepared on Standard English motion picture audits to order the record have been utilized. (C) In the third approach, a lexical resource called HindiSenti Word Net (H-SWN) have been built and a greater part score based technique to group the given archive have been executed. The outcomes for Hindi sentiment analysis support the distributed work that without such a corpus, MT-based frameworks give better characterization execution as looked at than dominant part construct frameworks situated in light of lexical resources. The three, in a specific order, constitute the fall-back strategy proposed for SA in Hindi. A possible assignment for the future concerning the resource based opinion investigation would be to join word sense disambiguation so that a particular feeling of word can be looked upward in the H-SWN. Another assignment would be to build another adaptation of H-SWN after the connecting of Hindi and English Word Net is finished. It is expected that the new form will have better scope.

M S et al. [5] has developed about sentiment analysis deals with identifying and classifying opinions or sentiments conveyed in source(reference) text. Sentiment analysis of twitter is difficult compared to general sentiment analysis due to the existence of slang texts and wrong spellings. The highest limit of characters that are allowed in Twitter is 140 . Knowledge formed outlook and Machine learning formed outlook are the two strategies used for analyzing sentiments from the text. The twitter posts about electronic products like mobiles, laptops ans son on using machine learning outlook in this paper are analyzed. By carrying out sentiment analysis in a specific domain, it is possible to identify the effect of domain data in sentiment categorization. A new feature vector for classifying the tweets as positive, negative and extract peoples' opinion about products is presented. Classification techniques were used in it: Nave bayes classifier, SVM classifier, Max entropy classifier, Ensemble classifier. Machine Learning techniques are simpler and efficient than symbolic techniques. These techniques can be applied for twitter sentiment analysis. There are certain issues while dealing with identifying emotional keyword from tweets having multiple keywords. It is also difficult to handle misspellings and slang words. To deal with these issues, an efficient feature vector is created by doing feature extraction in two steps after proper preprocessing. In the first step, twitter specific features are extracted and added to the feature vector. After that, these features are removed from tweets and again feature extraction is done as if it is done on normal text. These features are also added to the feature vector. Classification accuracy of the feature vector is tested using different classifier such as Ensemble, SVM, Naïve Bayes and Maximum Entropy classifiers. All these classifiers have almost similar accuracy for new attribute vector. The new attribute(feature) vector functions well for electronic products domain.

An improved NB classifier was proposed by Kang Hanhoon et al. [6] to solve the problem of the aptness for the positive categorization precision to present up to 10 percent approximately greater than the negative categorization precision. This produces an issue of decreasing the average precision when the precisions of the two categorizes are expressed as an average value. Utilizing the given algorithm with restaurant reviews reduced the space between the positive precision and the negative precision as compared with Naïve Bayes and SVM. The precision is upgraded in recall and precision compared to both NB and SVM.

It depicts the elements of a framework composed for the evaluation of movie reviews by Dziczkowski et al. [7]. Finally the system connects a numerical point automatically to every review; this is the objective of the system. It presents two new strategies in view of linguistic learning. Results are then contrasted and the general measurable system utilizing bayes classifier. The last step is to consolidate the outcomes got with a specific end goal to make the last appraisal as accurately as could be required under the situations. The framework exhibited does an accumulation of films reviews and automatically assigns a mark to each review. This system is a supporting for RS. The objective of our work is to robotize the entire framework, especially to allot a mark to individual client's surveys utilizing feeling discovery learning. The system permits a programmed task of a mark. Notwithstanding, to expand the examination on different fields it will be important to make a linguistic database and another investigation of the diverse components of the bunch's behavior. It succeeded in the creation and in the integration of two linguistic methodologies. This strategy made it possible to consequently assign a mark to the opinions in motion movie reviews.

Mao et al. [8] has explained the sentiment categorization has allured growing interest from natural language processing. The goal of sentiment categorization is to recognize automatically whether a specified piece of text expresses positive or negative opinion towards a subject of appeal. The standpoint have been presented that utilizes a human model based on random process to determine text polarity categorization is presented. Experiment outcomes displayed that on movie review corpus, the human modeling approach has a relatively greater precision than SVMs and Naïve bayes classifier. In the experiment, method to determine text polarity classification has many advantages (1) The method automatic extracts the semantic features without semantic word dictionary.(2) The accuracy of the method will be higher when more prior knowledge is added. In the future research, the sentiment features in the Sentence Similarity Computing will be applied. Sentence Similarity Computing is the most critical technology in QA system, and the sentiment of sentences influences the similarity of sentences.

\section{ALGORITHM IMPLEMENTATION}

A proposed algorithm implemented is discussed below:-

1. Enter the Punjabi text as input.

2. Divide this Punjabi paragraph into tokens and store the words in an array list.

3. Select the first word from array list.

4. Fetch the words of database in second array named as database array. 
5. Check whether selected paragraph word matched with each word of database array.

\section{(i) If match found}

(a) Find the sentiment of word from database whether it is positive/negative or neutral.

(b) Find the exact position of word in the paragraph.

(c) Highlight the word according to their sentiment; make it green if it is positive, red if it is negative and blue if it is neutral.

(d) Calculate the score of sentence.

(e) Store the results in database.

(ii) Else match not found

(a) Select next word from the array

(b) Go to step 5 .

6. Display the result to the user.

7. Plot the graph according to the results.

\section{RESULTS}

Sentiment analysis is a significant present research area. This introduces sentence classification using dictionary based approach for Punjabi language. The various techniques and systems available have been compared. The developed system finds out the positive, negative and neutral words from selected paragraphs.

\section{Example: 1}

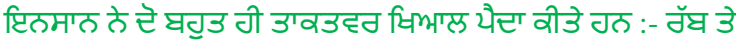

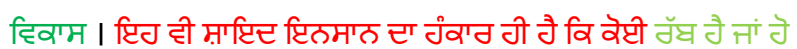
मवटा नै, ताले छि ही भा्टभी टे ग्रु हिष।

Negative word: गंवर्ठ

Positive Word: ठॅघ, टिवग्म

In this designed algorithm identified the negative word Jवर्ठ and highlights the sentence in red color according to their negative sentiment and Positive word ठॅघ, टिव'्म and highlights the sentence in green color according to their positive sentiment.

\section{Example: 2}

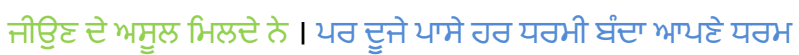

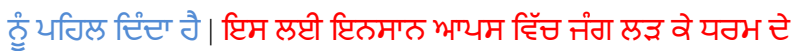
हां गेठ घंटे टा मंध्व वठटे के

Positive word: भमुल

Neutral word: घंटा, , घंटे

\section{Negative Word तंग}

In this the designed algorithm identified the negative word izr, positive word $n ;\{b$ and Neutral word घंटा , घंटे and highlights the sentence in red color according to their negative sentiment as well as in green color according to their positive sentiment and in blue color according to their neutral sentiment.

\section{CONCLUSION}

Sentiment Analysis is to differentiate and categorize the viewpoints or feelings or assessments in composed content. The feelings of the people can be expressed in positive, negative or neutral ways. Mostly, parts of speech are used as feature to extract the sentiment of the text. Sentiment analysis is an evolving field with a variety of use applications. Further, the accuracy of the system have been evaluated, from which it is analyzed that positive words are less used in reviews where as the number of negative words are more. The developed algorithm divide the sentence in three sentiments positive, negative and neutral to increase the accuracy of sentiment analyzing.

\section{FUTURE SCOPE}

The system built can be enhanced by adding provision for translating the Punjabi string into English or other dialect. Furthermore the current version of this algorithm does not facilitate the function of synonyms and antonyms but can be done in the future research processes.In future the developer or researcher use this algorithm for sentiment analyzing on regional language with the addition of disjunctive and synonym dictionary.

\section{REFERENCES}

[1] Karamibekr, M., \& Ghorbani, A. A. (2012). Sentiment analysis of social issues. Int. Conf. on Social Informatics, , 215-221.

[2] Kaur, A., \& Gupta, V. (Nov. 2013). A survey on sentiment analysis and opinion mining techniques. Proceedings of Journal of Emerging Technologies in Web Intelligence, Vol.5, No. 4.

[3] Phyu Shein, K. P., \& Nyunt, T. t. (2010). Sentiment Classification based on Ontology and SVM Classifier. Second International Conference on Communication Software and Networks.

[4] Joshi, A., A R, B., \& Bhattacharyya, P. (2010). A fall strategy for sentiment analysis in Hindi : A case study. International Conference on Natural Language Processing.

[5] M S, N., \& R, R. (2011). Sentiment Analysis in Twitter using Machine Learning Techniques- Sentiment analysis of Twitter data. LSM '11 Proceedings of the Workshop on Languages in Social Media, Association for Computational Linguistics, (pp.30-38).

[6] Kang Hanhoon, Y. S. (2012). Senti-lexicon and improved Naive Bayes algorithms for sentiment analysis of restaurant reviews. (pp. 39:6000-10). Expert Syst Appl.

[7] Dziczkowski, G., Wegrzyn-Wolska, K., \& Mines de Paris, E. d. (2008). An autonomous system designed for automatic detection and rating of film reviews. International Conference on Web Intelligence and Intelligent Agent Technology.

[8] Mao, J. (2012). Sentiment Classification Based on Random Process. International Conference on Computer Science and Electronics Engineering. 